\title{
Relation between trinucleotide GAA repeat length and sensory neuropathy in Friedreich's ataxia
}

\author{
L Santoro, G De Michele, A Perretti, C Crisci, S Cocozza, F Cavalcanti, M Ragno, \\ A Monticelli, A Filla, G Caruso
}

Department of Clinical Neurophysiology

L Santoro

A Perretti

\section{Department of \\ Neurology \\ G De Michele \\ A Filla \\ G Caruso}

Department of

Cellular Biology and

Pathology and CEOS,

Federico II University,

Naples, Italy

S Cocozza

F Cavalcanti

A Monticelli

Fondazione Clinica del Lavoro, Centro

Medico, Campoli MT

C Crisci

Ospedale Mazzoni, Ascoli, Italy

M Ragno

Correspondence to:

Dr Lucio Santoro, Servizio di

Neurofisiopatologia,

Università Federico II di

Napoli, Via Sergio Pansini 5, 80131 Napoli, Italy.

Telephone 0039817463103 ; fax 0039817462667 .

Received 17 March 1998 and in revised form

1 June 1998

Accepted 25 August 1998

\begin{abstract}
Objective-To verify if GAA expansion size in Friedreich's ataxia could account for the severity of sensory neuropathy.

Methods-Retrospective study of 56 patients with Friedreich's ataxia selected according to homozygosity for GAA expansion and availability of electrophysiological findings. Orthodromic sensory conduction velocity in the median nerve was available in all patients and that of the tibial nerve in 46 of them. Data of sural nerve biopsy and of a morphometric analysis were available in 12 of the selected patients. The sensory action potential amplitude at the wrist (wSAP) and at the medial malleolus (m mal SAP) and the percentage of myelinated fibres with diameter larger than 7,9 , and $11 \mu \mathrm{m}$ in the sural nerve were correlated with disease duration and GAA expansion size on the shorter (GAA1) and larger (GAA2) expanded allele in each pair. Pearson's correlation test and stepwise multiple regression were used for statistical analysis.

Results-A significant inverse correlation between GAA1 size and wSAP, $m$ mal SAP, and percentage of myelinated fibres was found. Stepwise multiple regression showed that GAA1 size significantly affects electrophysiological and morphometric data, whereas duration of disease has no effect.

Conclusion-The data suggest that the severity of the sensory neuropathy is probably genetically determined and that it is not progressive

(F Neurol Neurosurg Psychiatry 1999;66:93-96)
\end{abstract}

Keywords: Friedreich's ataxia, sensory neuropathy, GAA expansion

Friedreich's ataxia is the most frequent early onset autosomal recessive inherited ataxia. The disease is clinically characterised by progressive ataxia with onset before 20 years of age, absence of lower limb tendon reflexes, dysarthria, Babinski's sign, limb weakness, decreased vibration sense, and skeletal deformities. Cardiomyopathy and diabetes may also be found. ${ }^{1}$ Electrophysiological and pathological studies have suggested that axon degeneration and secondary demyelination constantly occur in peripheral sensory nerves. ${ }^{2-4}$

The gene causing the disease $(X 25)$ has been recently identified. ${ }^{5} X 25$ encodes a 210 amino acid protein with unknown function called "frataxin". Most patients (90\%) were homozygous for an unstable GAA trinucleotide expansion in the first intron of $X 25$. Normal chromosomes contained 7-22 GAA units, whereas Friedreich's ataxia chromosomes carried 200 to above 900 repeats. The remaining patients were heterozygous for the expansion and a few point mutations have been described in some of them. Advances in molecular genetics broadened the disease phenotype. In fact, the form with onset later than 20 years (late onset Friedreich's ataxia), ${ }^{6}$ and that with retained tendon reflexes ${ }^{7}$ are in linkage with the FRDA locus. Moreover, we recently described patients with minimal GAA expansion on one allele (ranging from 120 to 156 triplets) and without clinical and electrophysiological signs of sensory neuropathy. ${ }^{8}$

A clear relation between expansion size and phenotype variability has also been found. The expansion size inversely correlates with age at onset and directly with the presence of diabetes mellitus and cardiomyopathy. The best correlation was found with the size of the shorter allele in each pair. ${ }^{9-12}$

To verify that GAA expansion size could account for the severity of sensory neuropathy, we studied the relation between molecular data and peripheral nerve electrophysiological and pathological findings. We also investigated if disease duration might affect the severity of peripheral neuropathy.

\section{Patients and methods}

Fifty six patients were selected among 160 patients with Friedreich's ataxia examined since 1973 at the Department of Neurology of the Federico II University of Naples. The criteria of selection were homozygosity for GAA expansion and availability of electrophysiological findings. Forty three patients had typical Friedreich's ataxia, 11 had onset after 20 years of age, six had preserved tendon reflexes, four of them both late onset and pre- 
Table 1 Clinical, electrophysiological, and molecular findings

\begin{tabular}{|c|c|c|c|c|c|c|c|c|c|}
\hline & \multirow[b]{2}{*}{ Patients } & \multirow[b]{2}{*}{ Age } & \multirow{2}{*}{$\begin{array}{l}\text { Disease } \\
\text { duration }\end{array}$} & \multicolumn{2}{|c|}{ Wrist SAP amplitude } & \multicolumn{2}{|c|}{ m mal SAP amplitude } & \multirow[b]{2}{*}{$G A A 1$} & \multirow[b]{2}{*}{$G A A 2$} \\
\hline & & & & $\mu V$ & $\%$ & $\mu V$ & $\%$ & & \\
\hline \multicolumn{10}{|c|}{ Clinical, electrophysiological, and molecular findings: } \\
\hline Electrophysiological study & 56 & $23.8(13.7)$ & $8.3(9.2)$ & $1.32(1.62)$ & $10.2(15.6)$ & $0.36(0.42)$ & $18.3(27.3)$ & $697(227)$ & $905(143)$ \\
\hline Neuropathological study & 12 & $26.7(12.6)$ & $8.7(6.9)$ & $1.46(1.64)$ & $11.5(15.4)$ & $0.59(0.58)$ & $32.5(39.9)$ & $681(239)$ & $892(201)$ \\
\hline
\end{tabular}

Values are mean $(\mathrm{SD}) ; \mathrm{SAP}=$ sensory action potential; $\mathrm{m}$ mal=medial malleolus; control values: $\mathrm{n}=68$; age range $=15-44$ years; wrist $\mathrm{SAP}$ mean=14.3 $\mu \mathrm{V}$ (range $6-16$ ); $\mathrm{m}$ mal SAP mean $=2.16 \mu \mathrm{V}$ (range $0.2-3)$

served reflexes. Seven patients had diabetes mellitus wich had a mean onset of 28 (SD 9) years.

Orthodromic sensory conduction along the median and tibial nerves was studied using the technique described by Buchthal and Rosenfalck ${ }^{13}$ and by Behse and Buchthal. ${ }^{14}$ The sensory responses were led off via near nerve needle electrodes, and 250-2000 sweeps were electronically averaged. The surface temperature of the limb was kept constant at $37^{\circ} \mathrm{C}$. The median nerve results were available in all patients and that of the tibial nerve in 46 of them. The sensory action potential (SAP) amplitude along the median and tibial nerve was expressed either as an absolute value or as a percentage of the mean normal values of age matched controls from our laboratory.

A sural nerve biopsy and a morphometric analysis were performed in 12 of the selected patients with Friedreich's ataxia, who gave informed consent. A 7-8 $\mathrm{cm}$ specimen of sural nerve proximal to the lateral malleolus was excised totally under local anaesthesia. The fragments for light microscopy and morphometry were fixed in $2.5 \%$ glutaraldehyde in cacodylate buffer ( $\mathrm{pH} 7.4)$ at $4^{\circ} \mathrm{C}$ for 2 hours, postfixed in $2 \%$ osmium tetroxide in the same buffer for 2 hours, dehydrated in a graded alcohol series, and embedded in Epon 812. Sections of the whole nerve $(1 \mu \mathrm{m})$ were cut transversely, stained with $0.1 \%$ toluidine blue, and were used for the morphometric study. In each sample the outer diameter of at least 1000 myelinated fibres was redrawn on a planimeter with an electronic pen and measured with a computer assisted Kontron Videoplan system, using an "ad hoc" modified program of image analysis. The percentage of large myelinated fibres was also calculated.

The sensory action potential amplitude at the wrist (wSAP) and at the medial malleolus ( $\mathrm{m}$ mal SAP) and the percentage of myelinated fibres with diameters larger than 7,9 , and 11 $\mu \mathrm{m}$ in the sural nerve were correlated with disease duration and with GAA expansion size on the shorter (GAA1) and larger (GAA2) expanded allele in each pair.

Molecular analysis was performed according to previously described techniques. ${ }^{9}$

Relations between variables were studied by the Pearson's correlation test. For the stepwise multiple regression, SAP amplitudes and myelinated fibre percentages were the dependent variables and GAA1 and GAA2 triplet number, and disease duration were entered in order as predictive variables. The partial regression coefficient measures the expected change in the dependent variable when the predictive variable increases by 1 unit, the other predictive variables remaining unchanged.

\section{Results}

Clinical, electrophysiological, and molecular findings and the results of statistical analysis of all patients and of the 12 who underwent sural nerve biopsy are summarised in tables 1,2 , and 3.

ELECTROPHYSIOLOGICAL FINDINGS

We found a significant inverse correlation between GAA1 size and wSAP amplitude expressed either as an absolute value (figure) or

Table 2 Pearson's regression coefficients

\begin{tabular}{|c|c|c|c|c|c|c|c|}
\hline & \multicolumn{2}{|l|}{ Wrist SAP } & \multicolumn{2}{|c|}{ m. mal. SAP } & \multicolumn{3}{|c|}{ Percent of fibres } \\
\hline & $\mu V$ & $\%$ & $\mu V$ & $\%$ & $>7 \mu$ & $>9 \mu$ & $>11 \mu$ \\
\hline \multicolumn{8}{|c|}{ Pearson's regression coefficient: } \\
\hline GAA1 size & $-0.639^{\star \star \star \star}$ & $-0.660^{\star \star \star \star}$ & $-0.353^{\star}$ & $-0.570^{\star \star \star \star}$ & $-0.872^{\star \star \star}$ & $-0.802^{\star \star}$ & $-0.856^{\star \star \star}$ \\
\hline GAA2 size & -0.027 & -0.023 & -0.095 & -0.066 & -0.142 & 0.129 & 0.057 \\
\hline Disease duration & 0.214 & 0.257 & 0.098 & 0.279 & $0.779^{\star \star}$ & $0.672^{\star}$ & $0.690^{\star}$ \\
\hline
\end{tabular}

${ }^{\star} \mathrm{p}<0.05 ;{ }^{\star \star} \mathrm{p}<0.01 ;{ }^{\star \star \star} \mathrm{p}<0.001$

$\mathrm{SAP}=$ sensory action potential; $\mathrm{m}$ mal $=$ medial malleolus; control values: $\mathrm{n}=68$; age range $=15-44$ years; wrist $\mathrm{SAP}$ mean $=14.3 \mu \mathrm{V}$ (range 6-16); $\mathrm{m}$ mal SAP mean $=2.16 \mu \mathrm{V}$ (range $0.2-3$ ).

Table 3 Stepwise multiple regression:partial regression coefficients (SE)

\begin{tabular}{|c|c|c|c|c|}
\hline & \multicolumn{2}{|l|}{ Wrist SAP } & \multicolumn{2}{|l|}{ m mal $S A P$} \\
\hline & $\mu V$ & $\%$ & $\mu V$ & $\%$ \\
\hline \multicolumn{5}{|c|}{ Stepwise multiple regression: partial regression coefficients (SE) } \\
\hline GAA1 size & $-0.0051(0.0008)^{\star \star \star}$ & $-0.0503(0.0071)^{\star \star \star}$ & $-0.0006(0.0003)^{\star}$ & $-0.0695(0.0152)^{\star \star \star}$ \\
\hline GAA2 size & $0.0029(0.0012)^{\star}$ & $0.0301(0.0113)^{\star}$ & $0.0001(0.0005)$ & $0.0378(0.0248)$ \\
\hline Disease duration & $0.0249(0.0180)$ & $0.3139(0.1654)$ & $0.0013(0.0064)$ & $0.5417(0.3542)$ \\
\hline
\end{tabular}




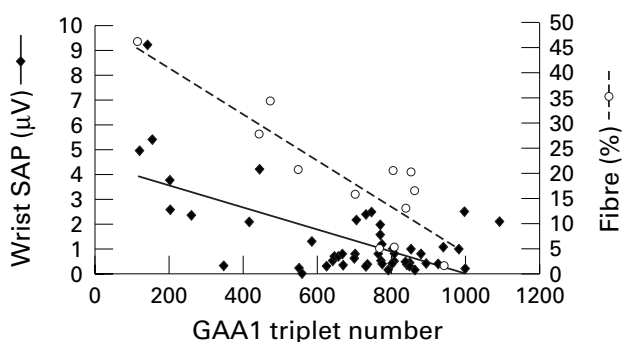

The continous line and the full symbols refer to the correlation between the number of GAA1 repeats and sensory action potential at the wrist (wSAP) in 56 patients $\left(r_{p}=-0.639 ; p<0.001\right)$. The dashed line and the open symbols refer to the correlation between the number of $G A A 1$ repeats and percentage of sural nerve fibres $>7 \mu \mathrm{m}$ in 12 patients $\left(r_{p}=-0.872 ; p<0.001\right)$.

as a percentage of the normal value. No correlation was found between GAA2 size and wSAP amplitude. There was a trend towards a direct correlation between disease duration and wSAP amplitude. We obtained similar results for the amplitude of $\mathrm{m}$ mal SAP (table 2).

Stepwise multiple regression of wSAP and $\mathrm{m}$ mal SAP amplitude on GAA1 and GAA2 size and disease duration showed that GAA1 size significantly affects neurophysiological findings. GAA2 size showed a slight significant effect only on wSAP amplitude. No effect of duration of disease was found (table 3).

\section{NEUROPATHOLOGICAL FINDINGS}

The mean value of the total number of the myelinated fibres in the sural nerve was 4361 (SD 1324) (normal 5662-10 732) and the mean percentages of the fibres larger than 7,9 , and $11 \mu \mathrm{m}$ were respectively 19.1 (SD 13.0) (normal 37.2-54.1), 9.1 (SD 9.6) (normal value 20.0-42.0), and 3.3 (SD 4.8) (normal 3.6-23.4).

Percentages of fibres $>7,9$, and $11 \mu \mathrm{m}$ were all directly correlated with duration of disease and inversely with GAA1 size (figure). Stepwise multiple regression showed that only GAA1 size was a significant determinant of large fibre loss.

\section{Discussion}

Friedreich's ataxia is the first autosomal recessive disease due to an expanded trinucleotide repeat. ${ }^{5}$ The discovery of the Friedreich's ataxia molecular defect has implications for the diagnostic criteria and for the explanation of the phenotypic variability of the disease. Filla et $a l^{9}$ showed that patients with late onset ataxia and preserved reflexes are homozygous for the GAA expansion, confirming the genetic homogeneity between typical Friedreich's ataxia and the atypical forms. The recent finding of

Table 3 (continued)

\begin{tabular}{lcc}
\hline Per cent of fibres & & \\
\hline$>7 \mu$ & $>9 \mu$ & $>11 \mu$ \\
\hline & & \\
$-0.0338(0.0108)^{\star \star \star}$ & $-0.0241(0.0083)^{\star \star}$ & $-0.0141(0.0038)^{\star \star \star}$ \\
$0.0106(0.0100)$ & $0.0208(0.0077)$ & $0.0086(0.0036)$ \\
$0.7776(0.3993)$ & $0.6007(0.3063)$ & $0.2488(0.1421)$ \\
\hline
\end{tabular}

patients homozygous for the expansion and with no signs of peripheral neuropathy ${ }^{8}$ prompted us to investigate the relation between the sensory neuropathy severity, as expressed by SAP amplitude, and the expansion size.

Loss of large peripheral nerve fibres is considered to be constant in Friedreich's ataxia, ${ }^{2-4}$ but there is no agreement about the pathogenesis of axon loss. The severity of myelinated fibre reduction seemed to increase with the patients' age,${ }^{15}$ but it was not related to disease duration or severity. ${ }^{4}{ }^{16}$ Said $e t ~ a l^{17}$ suggested a slow dying back degeneration of abnormally developed sensory neurons. We performed sural nerve biopsies and serial sensory nerve conduction studies in 15 patients with Friedreich's ataxia. ${ }^{18}$ In three of them a contralateral nerve biopsy was repeated after 6-7 years. Typical electrophysiological and pathological findings did not change with time, suggesting a very early but stable involvement of peripheral nerve, due to a defective development of the largest sensory neurons. Goto and Hirano ${ }^{19}$ suggested a selective involvement of these neurons by using immunohistochemical staining for substance $\mathrm{P}$ and synaptophysin. Frataxin expression is high in dorsal root ganglia (M Pandolfo, personal communication).

The present study shows a clear relation between expansion size and severity of sensory neuropathy. In fact, we showed an inverse correlation between the number of GAA1 repeats and neurophysiological (wSAP and $m$ mal SAP amplitude) and morphological findings (percentage of the largest fibres in the sural nerve). GAA1 size is the main factor determining severity of sensory neuropathy as also shown by multiple regression analysis. It accounts for $41 \%$ of the variation of wSAP amplitude and a difference in GAA1 size of 100 triplets determines a mean change of about $0.5 \mu \mathrm{V}$ in wSAP. The results of the analysis for sural nerve biopsy data were even more evident. GAA1 size accounts for $76 \%$ of variation of percentage of fibres $>7 \mu \mathrm{m}$. A difference in GAA1 size of 100 triplets determines a mean change of about $3.4 \%$ in fibre percentage.

The size of GAA2 is correlated neither with neurophysiological nor with morphological findings. However, multiple regression analysis showed a GAA2 effect on wSAP amplitude with higher amplitude values for higher GAA2 sizes. This effect, marginally significant $(\mathrm{p}<0.05)$ and biologically unlikely, may be due to chance.

In the group of patients with available peripheral nerve morphological findings, the number of remaining large fibres was inversely correlated with the size of GAA1 expansion and directly with duration of disease. An explanation of the last finding is that patients with lower GAA size had milder disease progression and underwent sural nerve biopsy later. There was a high inverse correlation between disease duration at biopsy and the number of repeats on GAA1 allele $\left(r_{\mathrm{p}}=-0.697\right.$; $\mathrm{p}<0.05$ ), and multiple regression analysis showed that duration of disease is not a significant determinant of the loss of large fibres. A trend towards a direct correlation between dis- 
ease duration and SAP amplitude was also found. However, multiple regression analyses showed that duration of disease does not significantly influence electrophysiological findings. On the other hand, a progression of the neuropathy during the disease course would have led to an inverse correlation between disease duration and both percentage of remaining large fibres and SAP amplitude. The presence of diabetes mellitus could affect the severity of the sensory neuropathy, although diabetes is a late feature in the course of the disease. We repeated the statistical analysis excluding the patients with diabetes and obtained similar results (data not shown).

Severe sensory neuropathy has been considered for a long time a hallmark of electrophysiology of patients with Friedreich's ataxia; however, the molecular diagnosis allowed us ${ }^{8}$ and others ${ }^{20}{ }^{21}$ to detect a few patients with Friedreich's ataxia without neuropathy. The present results show that very small expansions can be associated with very mild or absent sensory neuropathy, as measured by SAP amplitude. On the other hand, patients with an expansion above 600 GAA repeats have a full clinical expression of the involvement of peripheral nerves. This threshold is close to the 700 GAA repeats indicated by Dürr et $a l^{10}$ as the level above which the residual expression is too low to influence the clinical presentation. Our data suggest that the severity of the sensory neuropathy is probably genetically determined and that it is not progressive. Tissue mosaicism caused by mitotic instability, as has been shown in several triplet diseases including Friedreich's ataxia, ${ }^{11}$ might be a possible further mechanism that could explain the variability of severity of sensory neuropathy in patients with the disease. Studies on $X 25$ transcription and translation in the peripheral nervous system will elucidate this issue.

This work was partially supported by CNR-Rome (grant No 96.03274.CT04) to LS and Teletlou (grant No 969) to SC.

FC receives a fellowship from the Associazione Italiana per le Sindromi Atassiche
1 Geoffroy G, Barbeau A, Breton G, et al. Clinical description and roentgenologic evaluation of patients with Friedreich's ataxia. Can f Neurol Sci 1976;3:279-86.

2 Hughes JT, Brownell B, Hewer RL. The peripheral sensory pathway in Friedreich's ataxia. Brain 1968;91:803-18.

3 McLeod JG. An electrophysiological and pathological study of peripheral nerves in Friedreich's ataxia. 7 Neurol Sci 1971;12:333-49.

4 Caruso G, Santoro L, Perretti A, et al. Friedreich's ataxia: electrophysiological and histological findings. Acta Neurol Scand 1983;67:26-40.

5 Campuzano V, Montermini L, Moltò MD, et al. Friedreich's ataxia: autosomal recessive disease caused by an intronic GAA triplet repeat expansion. Science 1996;271:1423-7.

6 De Michele G, Filla A, Cavalcanti F, et al. Late onset Friedreich's disease: clinical features and mapping of mutation to
the FRDA locus. $\mathcal{F}$ Neurol Neurosurg Psychiatry 1994;57: the FR

7 Palau F, De Michele G, Vilchez JJ, et al. Early onset ataxia with cardiomyopathy and retained tendon reflexes maps to the Friedreich's ataxia locus on chromosome 9q. Ann Neurol 1995;37:359-62.

8 Ragno M, De Michele G, Cavalcanti F, et al. Broadened Friedreich's ataxia phenotype after gene cloning: minimal GAA expansion causes late onset spastic ataxia. Neurology GAA expansion caus

9 Filla A, De Michele G, Cavalcanti F, et al. The relationship between trinucleotide (GAA) repeat length and clinical features in Friedreich ataxia. Am 7 Hum Genet 1996;59: 554-60.

10 Dürr A, Cossée M, Agid Y, et al. Clinical and genetic abnormalities in patients with Friedreich's ataxia. $N$ Engl $\mathcal{F}$ Med 1996;335,16:1169-75.

11 Montermini L, Richter A, Morgan K, et al. Phenotypic variability in Friedreich ataxia: role of the associated GAA triplet repeat expansion. Ann Neurol 1997;41:675-82.

12 Filla A, De Michele G, Cocozza S. Genetic abnormalities in Friedreich's ataxia. N Engl f Med 1997;336:1021-2.

13 Buchthal F, Rosenfalck A. Evoked action potentials and conduction velocity in human sensory nerves. Brain Res 1966;3:1-122.

14 Behse F, Buchthal F. Normal sensory conduction in nerves of the leg in man. F Neurol Neurosurg Psychiaty 1971;34: 404-14.

15 Ouvrier RA, McLeod JG, Cochin TE. Friedreich's ataxia: early detection and progression of peripheral nerve abnormalities. F Neurol Sci 1982;55:137-45.

16 Caruso G, Santoro L, Perretti A, et al. Friedreich's ataxia: electrophysiologic and histologic findings in patients and relatives. Muscle Nerve 1987;10:503-15.

17 Said G, Marion MH, Selva J, et al. Hypotrophic and dyingback nerve fibers in Friedreich's ataxia. Neurology 1986;36: 1292-9.

18 Santoro L, Perretti A, Crisci C, et al. Electrophysiological and histological follow up study in 15 Friedreich's ataxia patients. Muscle Nerve 1990;13:536-40.

19 Goto S, Hirano A. Immunohistochemical evidence of the selective involvement of dorsal root fibres in Friedreich's ataxia. Neuropathol Appl Neurobiol 1990;16:365-70.

20 Klockgether T, Zühlke C, Schulz JB, et al. Friedreich's ataxia with retained tendon reflexes: molecular genetics, clinical neurophysiology, and magnetic resonance imaging. Neurology 1996;46:118-21.

21 Cruz-Martinez A, Anciones B, Palau F. GAA trinucleotide repeat expansion in variant Friedreich's ataxia families. Muscle Nerve 1997;20:1121-6. 